\title{
Lactoferrin suppresses the Epstein-Barr virus-induced inflammatory response by interfering with pattern recognition of TLR2 and TLR9
}

\author{
Ying Zheng ${ }^{1,2}$, Zailong Qin ${ }^{1}$, Qiurong Ye ${ }^{1}$, Pan Chen ${ }^{1,3}$, Zhen Wang ${ }^{4}$, Qun Yan ${ }^{5}$, Zhaohui Luo ${ }^{1}$, Xiaoping Liu ${ }^{1}$, \\ Yanhong Zhou' ${ }^{1,3}$, Wei Xiong ${ }^{1,3}$, Jian $\mathrm{Ma}^{1,3}$ and Guiyuan $\mathrm{Li}^{1,3}$
}

Epstein-Barr virus (EBV) infection contributes to tumorigenesis of various human malignancies including nasopharyngeal carcinoma (NPC). EBV triggers innate immune and inflammatory responses partly through Toll-like receptor (TLR) signaling. Lactoferrin (LF), with its anti-inflammatory properties, is an important component of the innate immune system. We previously reported that LF protects human B lymphocytes from EBV infection by its ability to bind to the EBV receptor CD21, but whether LF can suppress EBV-induced inflammation is unclear. Here, we report that LF reduced synthesis of IL-8 and monocyte chemoattractant protein-1 (MCP-1) induced by EBV in macrophages via its suppression of NF- $\kappa$ B activity. LF interacted with TLR2 and interfered with EBV-triggered TLR2-NF- $\kappa$ B activation. LF inhibited the ability of TLR9 to recognize dsDNA by binding to its co-receptor CD14, which blocked the interaction between CD14 and TLR9. EBV-induced inflammation was thus aggravated in the presence of CD14. In addition, $L F$ expression levels were significantly downregulated in NPC specimens, and correlated inversely with $I L-8$ and MCP-1 expression. These findings suggest that LF may suppress the EBV-induced inflammatory response through interfering with the activation of TLR2 and TLR9.

Laboratory Investigation (2014) 94, 1188-1199; doi:10.1038/labinvest.2014.105; published online 28 July 2014

Epstein-Barr virus (EBV) is a ubiquitous human gamma herpes virus that is associated with many diseases, such as Burkitt's lymphoma, Hodgkin's disease, and nasopharyngeal carcinoma (NPC). ${ }^{1}$ Although the major target cells for EBV infection are B lymphocytes, many other cell types have been reported to be permissive for $\mathrm{EBV}^{2-5}$ Increasing evidence indicates that monocytes or macrophages may have important implications in EBV pathogenesis. EBV infection modulates the profile of proinflammatory cytokines, including TNF- $\alpha$, IL- 6 , IL-8, and monocyte chemoattractant protein-1 (MCP-1), released by primary human monocytes. ${ }^{5-7}$ The inflammatory mediators triggered by EBV may be beneficial for preventing against infection at the early stage, but later may cause damage to the host, owing to unresolved inflammation, which might promote tumorigenesis. ${ }^{8,9}$
Toll-like receptors (TLRs) are key components of the innate immune system and sense certain structural motifs of microbes. ${ }^{10}$ Several reports have demonstrated that human TLRs can recognize EBV. $5,7,11,12$ Stimulation of human monocytes with either inactivated or infectious EBV virions leads to the release of MCP-1 and to an increase of several cytokine mRNA levels, including IL-8, through the engagement of the cell surface TLR2. ${ }^{7}$ More recently, TLR9 was found to contribute to the recognition of EBV DNA in primary monocytes, leading to an increased release of IL-8. ${ }^{5}$ Therefore, the inflammatory effect mediated by TLRs during the course of EBV pathogenesis is worthy of investigation.

Lactoferrin (LF; or lacotransferrin) is an iron-binding glycoprotein that is present in most biological fluids including human milk, tears, nasal secretions, saliva, intestinal

\footnotetext{
${ }^{1}$ Cancer Research Institute, Central South University, Hunan Key Laboratory of Nonresolving Inflammation and Cancer, Key Laboratory of Carcinogenesis, Ministry of Health, Key Laboratory of Carcinogenesis and Cancer Invasion, Ministry of Education, Changsha, China; ${ }^{2}$ Center for Medical Research, Second Xiangya Hospital, Central South University, Changsha, China; ${ }^{3}$ Hunan Provincial Tumor Hospital and the Affiliated Tumor Hospital of Xiangya School of Medicine, Central South University, Changsha, China; ${ }^{4}$ Diabetes Center, Second Xiangya Hospital, Institute of Metabolism and Endocrinology, Key Laboratory of Diabetes Immunology, Ministry of Education, Central South University, Changsha, China and ${ }^{5}$ Department of Clinical Laboratory, Xiangya Hospital, Central South University, Changsha, China Correspondence: Jian Ma, PhD or Guiyuan Li, PhD, Hunan Provincial Tumor Hospital and the Affiliated Tumor Hospital of Xiangya School of Medicine, Central South University, 283 Tongzipo Road, Changsha, Hunan 410013, China.
}

E-mail: majian@csu.edu.cn or lgy@csu.edu.cn

Received 3 February 2014; revised 17 June 2014; accepted 30 June 2014 
mucus, and genital secretions. LF is also synthesized and released by mucosal epithelia and neutrophils in response to inflammatory stimuli. Multiple biological functions have been described for LF, including iron homeostasis, antiinflammatory activity, immunomodulation, cancer protection, and antimicrobial activity. ${ }^{13-15}$ Although the cellular and molecular mechanisms accounting for the immunemodulatory effects of LF are far from being fully elucidated, it is now clear that the capacity of LF to influence cytokine production, either negatively or positively, relies, at least in part, on its ability to bind and sequester both lipopolysaccharide and its receptor CD14, as well as CpG DNA, thus preventing the downstream activation of pro-inflammatory pathways. ${ }^{16-18}$

Through the linkage analysis of 18 pedigrees from the Hunan province of South China, we previously revealed that potential susceptibility loci related to NPC are located on chromosome $3 \mathrm{p} 21 .{ }^{19}$ Further isolation and identification of a list of new tumor-suppressor genes for NPC from this region were pursued and $L F$ gene is one of the candidates. ${ }^{20}$ By means of cDNA microarray analysis, we found that LF mRNA levels were significantly downregulated in NPC tissues and that its expression levels were negatively correlated with the expression levels of inflammatory cytokines, such as IL-8 (unpublished data). More recently, we reported that LF could protect human primary B lymphocytes from EBV infection by binding directly to the EBV receptor $\mathrm{CD} 21$ on the B-cell surface. $^{21}$

In the present study, we evaluated the role of LF in the EBV-induced inflammatory response in macrophages and demonstrated that LF represses the EBV-induced inflammatory response by interfering with the pattern recognition of TLR2 and TLR9.

\section{MATERIALS AND METHODS Cell Cultures}

The human monocytic cell line THP-1 was cultured in RPMI 1640 medium supplemented with $10 \%$ fetal bovine serum. Human embryonic kidney (HEK) 293 cells were cultured in Dulbecco's modified Eagle's medium supplemented with $10 \%$ fetal bovine serum. To obtain macrophage-like differentiated THP- 1 cells (dTHP-1), THP-1 cells were treated with $25 \mathrm{ng} / \mathrm{ml}$ phorbol myristate acetate in the medium for $48 \mathrm{~h}$. After the incubation, the phorbol myristate acetate-containing medium was aspirated and adherent differentiated cells were resuspended in fresh medium and incubated for an additional $24 \mathrm{~h}$.

\section{Virus Purification and Viral DNA Isolation}

Purified EBV was obtained from the productive EBV B-cell lineage B95.8 as described previously. ${ }^{7}$ Viral titers were evaluated as described and expressed as transforming units per milliliter. ${ }^{7}$ Virus preparations were assayed for EBV genome content by quantitative PCR amplification for the Bam HI-W fragment. Viral dsDNA was purified from concentrated
EBV-B95.8 suspension with virus DNA extraction kit (Qiagen, Valencia, CA, USA) and the concentration was determined by spectrophotometry $(260 \mathrm{~nm})$.

\section{Plasmids}

Full-length LF- and TLR2-coding sequences (CDS) entirely lacking the 3'-UTR were purchased from GeneCopeia (Rockville, MD, USA) and subcloned into the eukaryotic expression vector pcDNA3.1 $(+)$ (Clontech, Mountain View, CA, USA). pcDNA3.1 $(+)$-hCD14 expression vectors were purchased from YRBIO (Changsha, China). PRL-TK and $\mathrm{NF}-\kappa \mathrm{B}$ luciferase reporter plasmids were purchased from Invitrogen (Carlsbad, CA, USA). To construct deletion LF mutant vector, we deleted the 2-34 amino acids in the $\mathrm{N}$-terminal of LF protein so as to abrogate its ability of interacting with CD14. ${ }^{17}$ The LF or CD14 expression vectors were transfected into dTHP-1 cells. Forty-eight hours post transfection, cells were stimulated with infectious EBV, EBV DNA, or CpG-2006.

\section{Cell Treatment and Quantitative Real-Time RT-PCR (qRT-PCR)}

Human LF (hLF) proteins and human transferrin (hTF) proteins were obtained from Sigma-Aldrich (St Louis, MO, USA), which are iron free. Chloroquine (CQ), a known inhibitor of endosomal acidification, was also purchased from Sigma-Aldrich. Human-specific type B CpG ODN Oligodeoxynucleotides (ODN) 2006 (5'-tcgtcgttttgtcgttttgtcgtt- $\left.3^{\prime}\right)$ were resuspended in endotoxin-free water and used as positive control for TLR9 activation at the concentration as described in the literature (lowercase letters are phosphorothioate linkage). The oligonucleotides were synthesized by Sangon Biotech (Shanghai, China). For all hLF treatment, hLF or hTF was added into dTHP-1 cells $1 \mathrm{~h}$ before EBV infection or EBV DNA or CpG-2006 stimulation, and the hLF was present during EBV infection or EBV DNA or CpG DNA treatment.

Total RNA from the cells or tissues was isolated using Trizol (Invitrogen), and cDNA was synthesized from $1 \mu \mathrm{g}$ of total RNA by means of the reverse reaction kit, according to the manufacturer's instructions (Promega, Madison, WI, USA). And then quantitative real-time RT-PCR analysis of $I L-8$ and $M C P-1$ was performed in an iQ5 qRT-PCR detection system (Bio-Rad, Hercules, CA, USA). All reported results are the average ratios of three different independent experiments. Primer sequences for quantitative PCR: $I L-8$ forward $5^{\prime}$-ACTCCAAАССТTТCСАCC- ${ }^{\prime}$, reverse $5^{\prime}$-AAC TTC TCCACAACCCTC- $3^{\prime}$. MCP-1 forward $5^{\prime}$-CATTGTGG CCAAGGAGATCTG- ${ }^{\prime}$, reverse $5^{\prime}$-CTTC GGAGTTTGGG TTTGCTT- $3^{\prime}$. GAPDH forward 5'-ATCAAGATCATTGCTCC TCCTGAG- ${ }^{\prime}$, reverse 5'-CTGCTTGCGATCCACATCTG-3' . All the nasopharyngeal tissue samples were obtained from the Xiangya Hospital (Changsha, China), with written informed consent from patients and with approval for experiments 
from the ethical review committees of the appropriate institution. See Table 1 for patients' clinicopathologic features.

\section{ELISA}

Cell-free supernatants were harvested at indicated times after stimulation for IL-8 and MCP-1 quantifications by ELISA according to the manufacturer's protocol (Cusabio, China).

\section{SiRNA Transfection}

The pre-designed siRNAs against LF and CD14 used in this manuscript were purchased from GenePharma (Shanghai, China). The dTHP- 1 cells were seeded at $1 \times 10^{6}$ cells per well in a six-well plate. The next day, cells were transfected with $100 \mathrm{nM}$ siRNAs by using Lipofectamine 2000 (Invitrogen) for $6 \mathrm{~h}$. Medium was then replaced, and cells were kept in culture for an additional $42 \mathrm{~h}$ before stimulation.

\section{Luciferase Assay}

HEK293 cells were cotransfected with selected expression plasmids using Lipofectamine 2000 along with NF- $\kappa$ B luciferase reporter plasmid. Briefly, cells were seeded at $5 \times 10^{4}$ cells per well in a 24 -well plate $14 \mathrm{~h}$ before transfection. Forty-eight hours post transfection, cells were infected with EBV (MOI of 1.0) for the indicated times. Following stimulation, cells were lysed in luciferase buffer. Luciferase activity was measured by luminometry, and relative light units were normalized by Renilla luciferase.

\section{Confocal Microscopy}

To observe the cellular localization of LF and TLR2 proteins, dTHP-1 cells were cultured on coverslips and incubated with

\section{Table 1 Clinicopatholocial features of the NPC patients}

\begin{tabular}{lc}
\hline Viable & Number of cases \\
\hline Gender & 13 \\
Male & 5 \\
Female & \\
Age (years) & 3 \\
$\geq 60$ & 15 \\
$<60$ & \\
TNM stage & \\
Stage I or II & 4 \\
Stage $\geq$ III & 14 \\
& \\
Lymph node status & \\
Metastasis & 15 \\
No metastasis & 3 \\
\hline
\end{tabular}

hLF $(2.5 \mu \mathrm{g} / \mathrm{ml})$ for $1 \mathrm{~h}$. After $1 \mathrm{~h}$, cells were fixed and then incubated with anti-LF (Santa Cruz Biotechnology, Santa Cruz, CA, USA) and TLR2 antibodies (Epitomics, Burlingame, CA, USA). After washing with PBS, cells were incubated with Alex Flour 488-conjugated anti-mouse IgG (target LF antibody) and Alex Flour 568-conjugated anti-rabbit IgG antibody (target TLR2 antibody) and then observed by confocal microscope (FluoView FV1000 Confocal Microscope, Olympus, Tokyo, Japan). The merged image represents the extent of the colocalization of LF with TLR2 in the cell. The TLR9 activation was determined by observing intracellular trafficking of TLR9 and CD63, which is the late endosome marker (mouse antihuman CD63, clone MEM-259; Biolegend, San Diego, CA, USA) detected by a FITC-conjugated antimouse IgG (Beyotime, Jiangshu, China). TLR9 labeling was done with a rat antihuman TLR9 (clone eB72-1665; eBioscience, San Diego, CA, USA) followed by a Cy5-conjugated antirat IgG (Beyotime). The co-location of TLR9 and CD14 was determined with a rat antihuman TLR9 (eBioscience) and a mouse antihuman CD14 (Santa Cruz Biotechnology), then labeled by a Cy5-conjugated antirat IgG and a FITC-conjugated antimouse IgG.

\section{Immunoblotting}

Western blotting was carried out as described previously. ${ }^{20}$ Nuclear protein was isolated with nuclear and cytoplasmic Extraction Kit (Thermo Fisher Scientific, Waltham, MA, USA). Antibodies against p65, GAPDH and CD14 were obtained from Santa Cruz Biotechnology.

\section{Co-Immunoprecipitation}

To assay the interaction between LF and TLR2 protein, the dTHP- 1 cells were incubated with hLF $(2.5 \mu \mathrm{g} / \mathrm{ml})$ at $4{ }^{\circ} \mathrm{C}$ for $1 \mathrm{~h}$. The hLF-treated cells were lysed in modified immune precipitation buffer, and insoluble material was removed by centrifugation. Anti-LF antibody (Santa Cruz Biotechnology) or anti-TLR2 antibody (Epitomics) was then added to the lysates, incubated overnight at $4{ }^{\circ} \mathrm{C}$. A respective unrelated IgG antibody was used as immunoprecipitation control. The next day, immune complexes were bound to protein A/Gagarose beads (Merck Calbiochem, Darmstadt, Germany) for $1 \mathrm{~h}$ at $4{ }^{\circ} \mathrm{C}$. Immunobeads were washed four times with lysis buffer, and the precipitates were resuspended in SDS sample buffer. The immunoprecipitates were resolved on SDS-PAGE followed by western blot analysis with anti-TLR2 or LF antibodies. The similar processes were performed to prove the interaction of LF and CD14. The antibodies against LF and CD14 used in the experiment were obtained from Epitomics and Santa Cruz Biotechnology, respectively. To evaluate the effect of LF on the ability of binding of TLR9 to CD14, the dTHP-1 cells were treated with hLF $(50 \mu \mathrm{g} / \mathrm{ml})$ or not. Then, the cells were stimulated with EBV DNA $(10 \mu \mathrm{g} / \mathrm{ml})$ or CpG-2006 $(10 \mu \mathrm{g} / \mathrm{ml})$. After $20 \mathrm{~min}$, the cells were lysed in modified immune precipitation buffer and were then precipitated with anti-TLR9 or CD14 antibodies. The 
immunoreactivity was determined with anti-CD14 antibodies or TLR9 antibodies.

\section{Statistical Analysis}

Spearman's correlation test was used to evaluate the pairwise association of specimens' $L F$ expression levels and $I L-8$, $M C P-1$ levels. Student's $t$-test is used to compare treatment to controls. Two-way ANOVA is used to compare the difference among three or more experiment groups. Calculations were performed using the SPSS 15.0 statistical software. $P<0.05$ is considered statistically significant.

\section{RESULTS}

\section{LF Inhibits EBV-Induced Upregulation of MCP-1 and IL-8} in Macrophages

TLR engagement following EBV stimulation results in a rapid production of inflammatory cytokines and antiviral mediators, such as MCP-1 and IL-8. LF has inhibitory effects on inflammatory responses triggered by bacterial or viral components, so in this study, we first assessed whether LF may inhibit the synthesis of MCP-1 and IL- 8 induced by EBV infection in macrophages. The dTHP-1 were pre-incubated with various concentrations of hLF $(0-100 \mu \mathrm{g} / \mathrm{ml})$ or $100 \mu \mathrm{g} / \mathrm{ml}$ hTF (as control), and then stimulated with EBV virions. As shown in Figure 1a, EBV infection induced a significant upregulation of MCP-1 and IL-8 mRNA and protein expression levels in the macrophages, whereas upregulation was reduced by hLF but not by hTF. EBV infection-induced $M C P-1$ and $I L-8$ upregulations were also repressed significantly by transfection of a LF expression vector (Figure 1b). To further confirm the inhibitory effects of LF on EBVinduced inflammatory responses in macrophages, we used siRNA to inhibit endogenous LF expression (see Figure 1c), and found that EBV-induced $M C P-1$ and $I L-8$ expression were increased by $37 \%$ upon LF knockdown (Figure 1d). These observations suggest that LF may inhibit EBV infection-induced MCP-1 and IL-8 upregulation.

\section{LF Represses TLR2-Dependent NF- $\kappa$ B Activation Induced by EBV}

Because EBV infection may lead to the release of MCP-1 and IL-8 in an TLR2-NF- $\kappa$ B-dependent manner, ${ }^{7}$ we next determined the effect of LF on EBV-induced TLR2-NF- $\kappa$ B activation. The dTHP-1 cells were transfected with LF-expression vector or control vector. Forty-eight hours post transfection, cells were stimulated with infectious EBV. Following stimulation, the expression levels of nuclear NF- $\kappa \mathrm{B}$ (p65) protein were determined. Figure 2a showed that EBV infection stimulated nuclear NF- $\kappa \mathrm{B}$ expression, whereas LF transfection partially reduced this effect. We then determined the effect of LF and TLR2 transfection on EBV-induced NF- $\kappa$ B activation through a NF- $\kappa$ B luciferase reporter assay. LF, TLR2 expression vector, NF- $\kappa$ B luciferase reporter plasmids were co-transfected into HEK293 cells, followed by stimulation of EBV infection (either 8 or $24 \mathrm{~h}$ ). As Figure $2 \mathrm{~b}$ showed, TLR2 transfection increased EBV-induced NF- $\kappa$ B luciferase activity by $86 \%$, whereas LF transfection partly reversed this increase. These results indicate that TLR2 has an important role in EBV-induced NF- $\kappa$ B activation, and LF can repress EBV-induced TLR2-dependent NF- $\kappa$ B activation.

\section{LF Binds to TLR2}

Owing to its net positive charge, LF can bind to many types of microorganisms and proteins. ${ }^{16,17}$ We proposed that LF could interact with TLR2 on macrophages, and thus disturb EBV binding to TLR2. In an initial experiment, we used immunofluorescence assay to visualize the location of LF and TLR2. For visualization of LF, hLF was added to the dTHP-1 cells for $1 \mathrm{~h}$ and then washed away. LF and TLR2 were observed to colocalize on the cell surface and cytoplasm at a certain extent (Figure 3a). We then performed co-immunoprecipitation experiments to evaluate these two proteins' binding ability. hLF was added to the cells for $1 \mathrm{~h}$ and then washed away, then cell lysates were immunoprecipitated with either anti-TLR2 or anti-LF antibody. Figure $3 b$ showed that LF can bind to TLR2 protein. This observation implies that the inhibitory effect of LF on EBV-induced NF- $\kappa$ B activation may be due to its interaction with TLR2 of macrophages.

\section{LF Suppresses the Activation of TLR9 followed by EBV DNA Stimulation}

TLR9 is a receptor for nucleic acid-containing unmethylated CpG motifs, which is present in both bacterial and viral DNA. EBV DNA activates TLR9, thus leading to the release of IL-8 by monocytes. ${ }^{5}$ As we have observed that LF could suppress the production of IL-8, we investigated whether LF has an inhibitory role in the EBV DNA-TLR9-IL-8 pathway. To address this question, the dTHP-1 cells were stimulated with EBV DNA following pretreatment with LF or CQ (a known inhibitor of TLR9 activation), ${ }^{5}$ and then the expression levels of $I L-8$ were determined. CpG-2006 ODN is a potent TLR9 activator that induces cytokine secretion ${ }^{22}$ and was used as a positive control in this study. EBV DNA or CpG-2006 stimulation induced a significant increase of IL-8 expression and secretion, but this induction was suppressed significantly by hLF pretreatment, and the production of IL-8 in EBV DNA or CpG-2006-stimulated macrophages was abolished by CQ, as expected (Figure 4a). TLR9 is mainly expressed in the endoplasmic reticulum of resting immune cells. Following EBV DNA stimulation, a rapid migration of TLR9 toward early endosomes is initiated, and then a subsequent fusion of those vesicles with lysosomes promotes the formation of late endosomes and their maturation. The redistribution of TLR9 in late vesicle compartments represents its functional activation. ${ }^{5}$ The dTHP- 1 cells were treated with hLF, followed by stimulation of EBV DNA or CpG-2006, and then stained for TLR9 and CD63 (a marker for late endosomes). Colocalization of TLR9 and CD63 was observed following only 15-30 min EBV DNA or CpG-2006 stimulation, whereas LF pretreatment significantly inhibited 

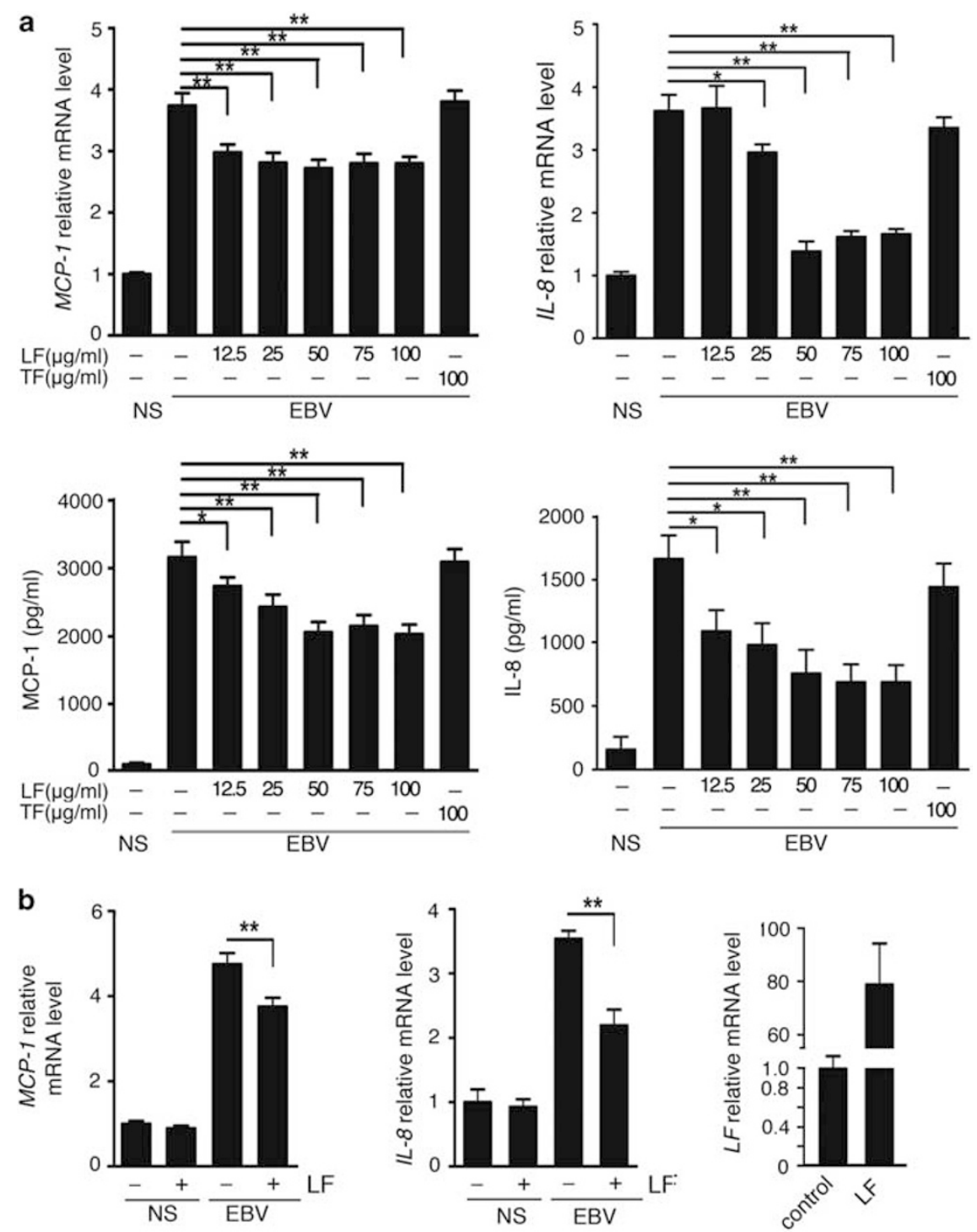

C

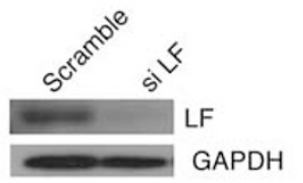

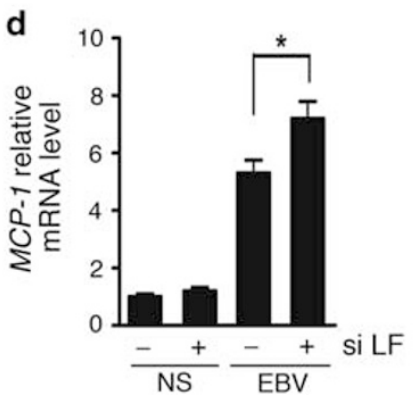

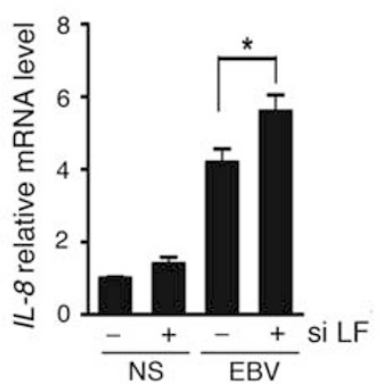

Figure 1 LF represses EBV-induced MCP-1 and IL-8 expression and secretion. (a) The dTHP-1 cells were treated with hLF (0-100 $\mu \mathrm{g} / \mathrm{ml})$ or hTF $(100 \mu \mathrm{g} / \mathrm{ml}$, as control), followed by EBV infection $(\mathrm{MOI}=1.0)$. The mRNA and protein expression levels of MCP-1 and IL-8 were determined by qRT-PCR (upper panel) and ELISA (lower panel). (b) The dTHP-1 cells were transiently transfected with LF expression vector or control vector. Forty-eight hours post transfection, cells were stimulated with infectious EBV $(\mathrm{MOI}=1.0)$ and $10 \mathrm{~h}$ followed by qRT-PCR assay to assay the $M C P-1$ and $I L-8$ expression levels. LF mRNA levels were also determined to evaluate LF expression vector efficiency. (c) The dTHP-1 cells were transfected with siRNA-targeting LF or scrambled siRNA. Forty-eight hours after transfection, total protein was extracted and western blotting was performed using antibodies against LF and GAPDH. (d) The dTHP-1 cells were pretreated with LF siRNA or scrambled siRNA, followed by further stimulation with infectious EBV (MOI = 1.0). $M C P-1$ and IL-8 expression were determined by qRT-PCR. ${ }^{*} P<0.05 ;{ }^{*} P<0.01$, compared with respective control. NS, not stimulated. 
a
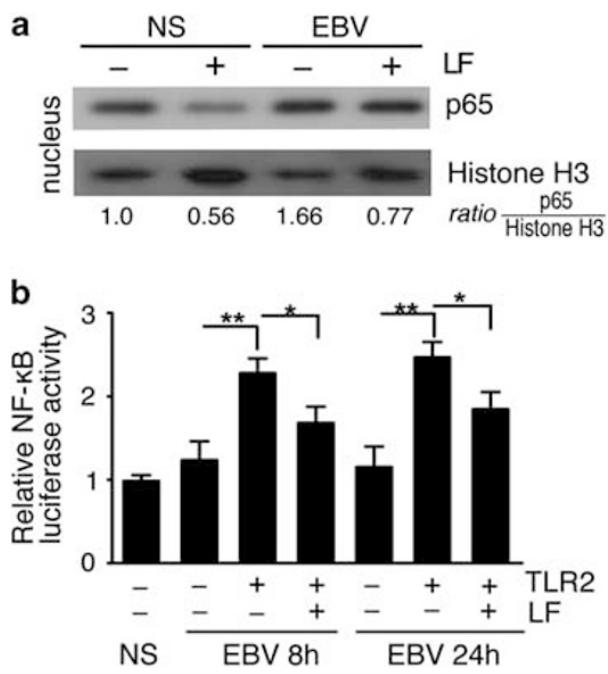

Figure 2 LF inhibits NF- $\kappa$ B activation. (a) dTHP-1 cells were transfected with LF expression vector or control vector, followed by stimulation of $\mathrm{EBV}(\mathrm{MOI}=1.0)$. One hour post stimulation, the nuclear protein was extracted and the expression levels of p65 were determined. Histone H3 was used as loading control. Relative accumulation of p65 proteins in the various groups is indicated. (b) HEK293 cells were transiently transfected with TLR2 and NF- $\kappa$ B luciferase reporter plasmid. Forty-eight hours post transfection, cells were stimulated with infectious EBV $(\mathrm{MOI}=1.0)$ for 8 or $24 \mathrm{~h}$, and luciferase assay was performed. ${ }^{*} P<0.05 ;{ }^{*} P<0.01$, compared with respective samples as indicated. NS, not stimulated.
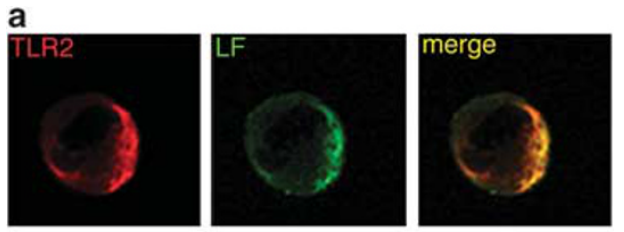

b

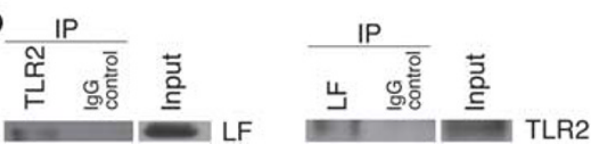

Figure 3 LF interacts with TLR2. (a) Immunofluorescence colocalization of LF with TLR2. The dTHP-1 cells were incubated with hLF $(2.5 \mu \mathrm{g} / \mathrm{ml})$ for $1 \mathrm{~h}$. After washing, bound LF was detected by anti-LF antibody (green), and TLR2 was detected by anti-TLR2 antibody (red). The cells were observed using confocal fluorescent microscopy. The yellow color in the merged image represents the extent of the colocalization of LF with TLR2. (b) Co-immunoprecipitation of LF with TLR2. The dTHP-1 cells were treated with $\mathrm{hLF}(2.5 \mu \mathrm{g} / \mathrm{ml})$ for $1 \mathrm{~h}$. After washing, the cell lysates were subjected to co-immunoprecipitation with either anti-TLR2 or anti-LF antibodies (an unrelated IgG antibody was used as negative immunoprecipitation (IP) control). Immunoprecipitates were analyzed by western blot with anti-LF or anti-TLR2 antibodies.

the redistribution of TLR9 in late endosomes (Figure 4b). The results indicate that LF may inhibit the activation of TLR9, thereby interfering with IL-8 induction by EBV DNA.

\section{CD14 is Involved in EBV or EBV DNA-Induced IL-8 Expression in Macrophages}

TLRs require additional proteins to be activated by their respective ligands. CD14 has been recently reported as necessary for TLR7- and TLR9-dependent induction of proinflammatory cytokines. CD14 can function as the co-receptor of TLR9, and promote its activation induced by viral DNA. ${ }^{23}$ Here, we investigated whether CD14 was implicated in EBV-induced cytokine secretion. The dTHP-1 cells were transfected with CD14 expression vector, followed by EBV infection or CpG-2006 stimulation, and then the expression levels of $I L-8$ were determined. EBV- or CpG2006-induced IL-8 upregulation was enhanced by CD14 transfection (Figure 5a). We then used CD14-targeting siRNA to suppress CD14 expression (Figure 5b), and found that knockdown of endogenous CD14 can abolish the IL-8 induction upon EBV or CpG2006 stimulation (Figure 5c). To evaluate the contribution of CD14 to the TLR9-dependent production of IL-8, dTHP-1 cells transfected with the CD14 expression vector were stimulated with EBV DNA, and IL-8 expression and secretion were determined. We observed that CD14 enhanced EBV-DNA-induced IL-8 upregulation and secretion (Figure 5d). As a control, CQ (an inhibitor of TLR9 activation) totally reversed IL-8 induction under the same situation (Figure 5d). These results demonstrate that CD14 is essential for EBV or EBV DNA-induced release of IL-8 by macrophages.

\section{LF Negatively Regulates TLR9 Pathway through Binding and Inhibiting CD14}

LF inhibits inflammatory cytokine secretion induced by CD14-lipopolysaccharide complex through its interaction with CD14. ${ }^{16,17}$ As the co-receptor of TLR9, CD14 may increase the production of IL-8 induced by EBV DNA. Therefore, we considered that the interaction between LF and CD $14^{17}$ may be involved in the inhibition of EBV-DNAinduced IL-8 production by LF. First, we took advantage of co-immunoprecipitation assays to confirm binding of LF and CD14. Then, hLF was added to the dTHP-1 cells and lysates were immunoprecipitated with either anti-CD14 or anti-LF antibody. Figure 6a showed that LF can bind to CD14 protein. The dTHP-1 cells were co-transfected with LF and CD14 expression vectors, followed by EBV DNA stimulation, and then $I L-8$ expression levels were determined. LF suppressed the CD14 promotion of EBV-DNA-induced $I L-8$ upregulation (Figure $6 \mathrm{~b}$ ). We also found that hLF pretreatment led to a partial repression of CD14-enhanced IL-8 upregulation induced by EBV DNA stimulation (Figure 6c). To confirm that the inhibitory effect of LF is dependent on the binding of LF and CD14, we constructed a deletion mutant of LF, which cannot bind to CD14. The LF expression vector or LF mutant vector was transfected into the cells with CD14 expression vector, followed by EBV DNA treatment. We observed that LF partially inhibited the effects of CD14 on IL-8 induction, whereas the LF deletion mutant did not have this ability 

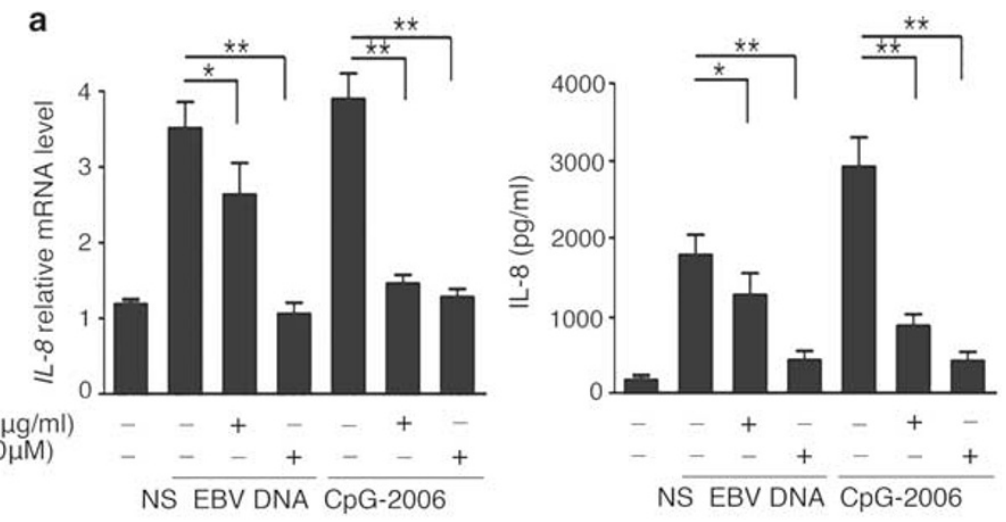

\section{b}
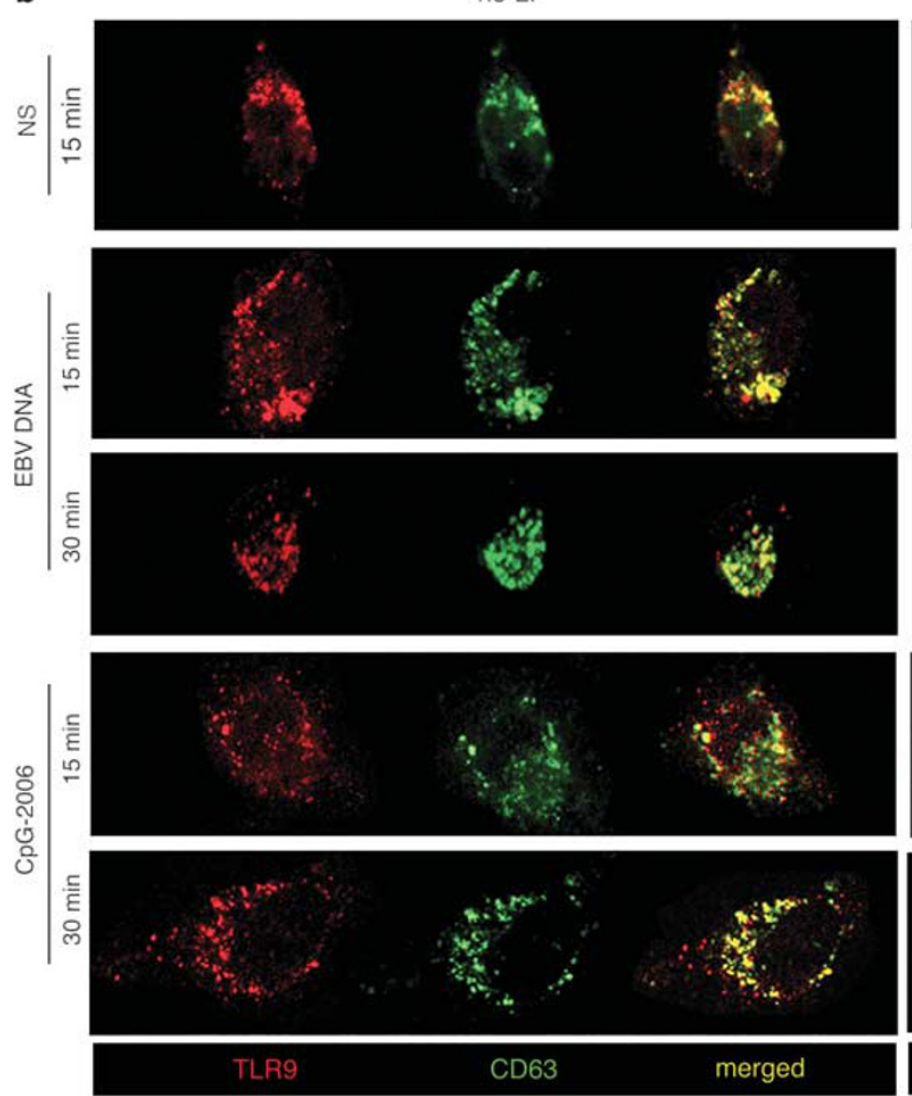
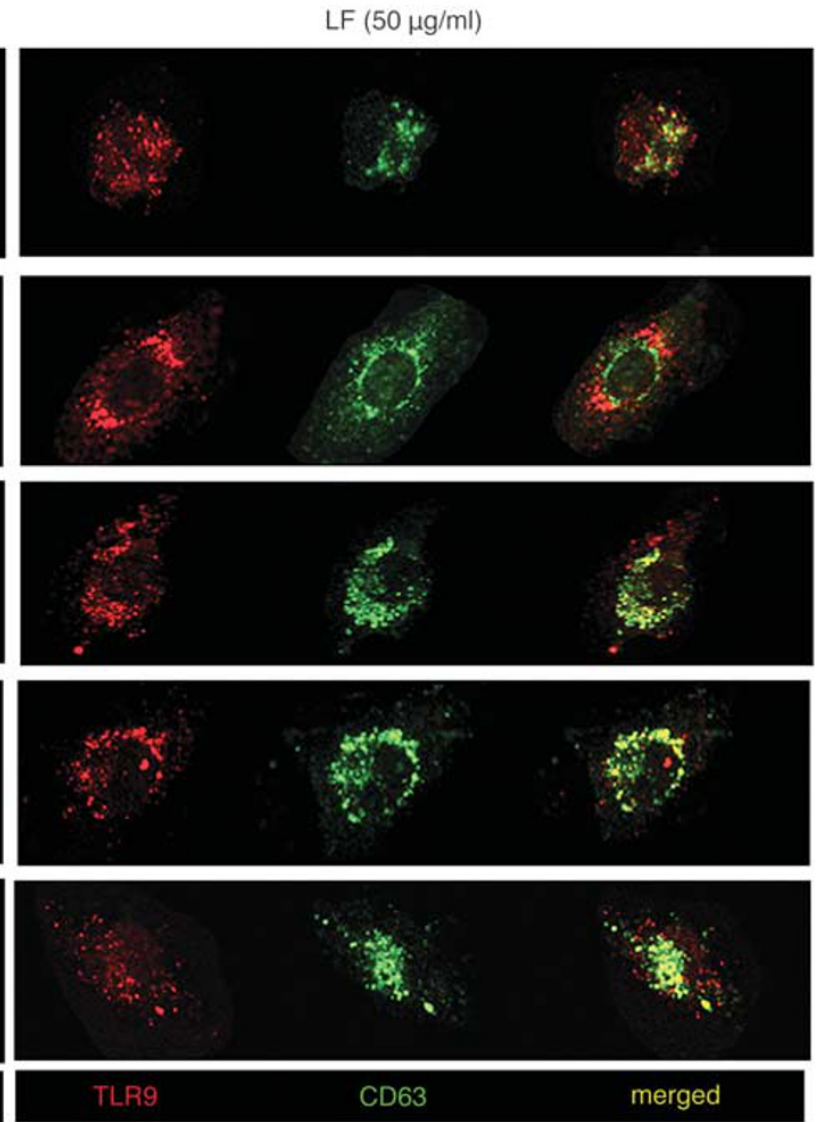

Figure 4 LF inhibits TLR9 activation and IL-8 upregulation. (a) dTHP-1 cells were stimulated with EBV DNA (10 $\mu \mathrm{g} / \mathrm{ml})$ or CpG-2006 (10 $\mu \mathrm{g} / \mathrm{ml}$, as a positive control for TLR9 activation) for $10 \mathrm{~h}$, and the total RNA and cell-free supernatants were harvested for IL-8 determinations by qRT-PCR (left panel) and ELISA (right panel). Where indicated, cells were pre-treated with CQ ( $20 \mu \mathrm{M}$, a known inhibitor of TLR9 activation) or LF (50 $\mu \mathrm{g} / \mathrm{ml})$ for $1 \mathrm{~h}$ before stimulation. ${ }^{*} P<0.05$; ${ }^{* *} P<0.01$, compared with respective control. NS, not stimulated. (b) LF blocked TLR9 recruitment within late endosomal compartments induced by EBV DNA. dTHP-1 cells were treated or not with hLF ( $50 \mu \mathrm{g} / \mathrm{ml})$ for $1 \mathrm{~h}$, followed by stimulation of EBV DNA, or CpG-2006 $(10 \mu \mathrm{g} / \mathrm{ml})$. TLR9 expression and colocalization with late endosome were visualized by confocal microscopy using double labeling of cells with anti-TLR9 (Cy-5 red) and anti-CD63 (FITC, green, a marker for late endosomes) antibodies.

(Figure 6d). Next, we assayed the effect of LF on the binding of CD14 to TLR9. The dTHP-1 cells were stimulated with EBV or CpG-2006, following hLF pretreatment. Confocal microscopic analysis showed a rapid colocalization of TLR9 and CD14 proteins in the cells after EBV infection or
CpG-2006 stimulation, which indicated that CD14 is engaged in the recognition of EBV by TLR9. However, in the cells with LF pretreatment, the colocalization appeared suppressed compared with the levels in untreated cells (Figure 6e). We also used co-immunoprecipitation experiments to observe 
the effect of LF on the binding of CD14 and TLR9 induced by EBV DNA. The dTHP-1 cells were treated with hLF $(50 \mu \mathrm{g} / \mathrm{ml})$ or not. Then, the cells were stimulated with EBV DNA or CpG-2006. After $20 \mathrm{~min}$, the cells were collected, and the co-immunoprecipitation experiment was performed with anti-CD14 antibodies or TLR9 antibodies. Figure 6f shows that EBV DNA or CpG-2006 stimulation enhances the binding of CD14 and TLR9, whereas LF attenuates it. These

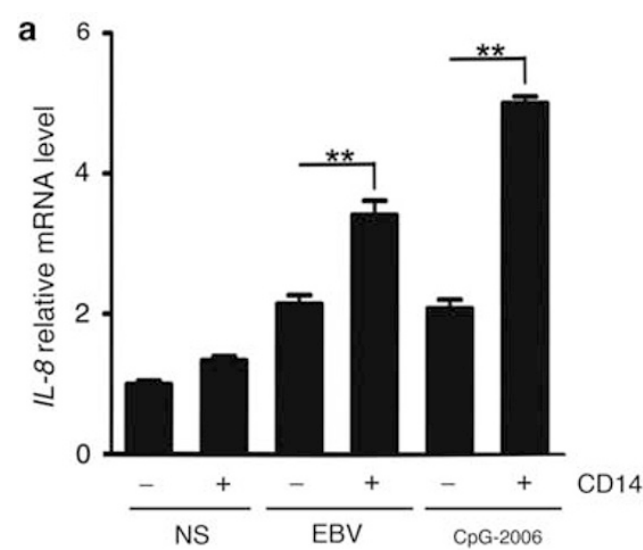

d

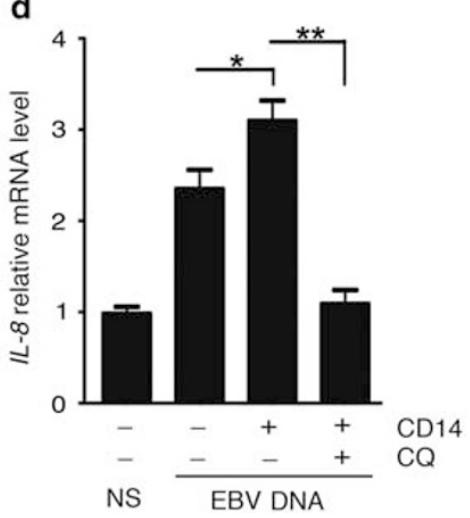

b

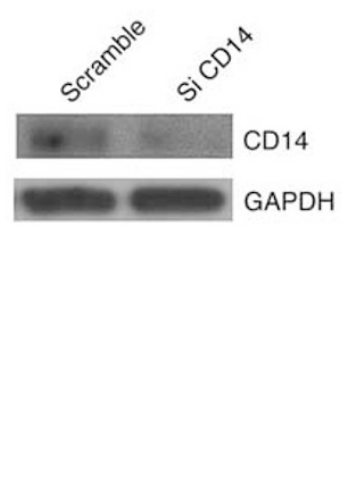

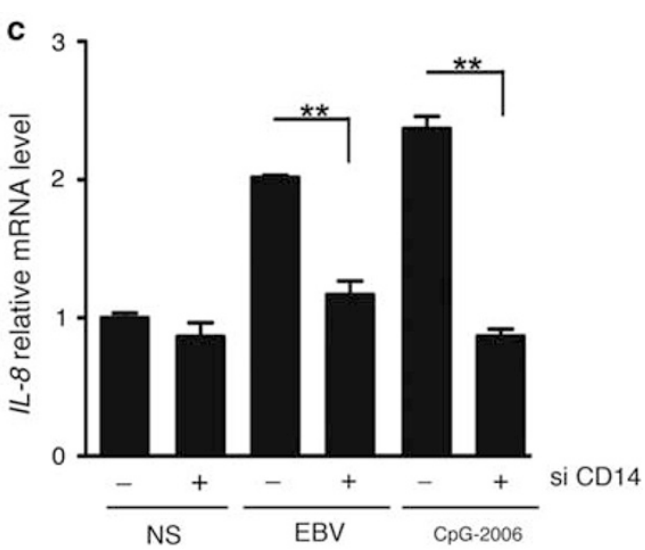

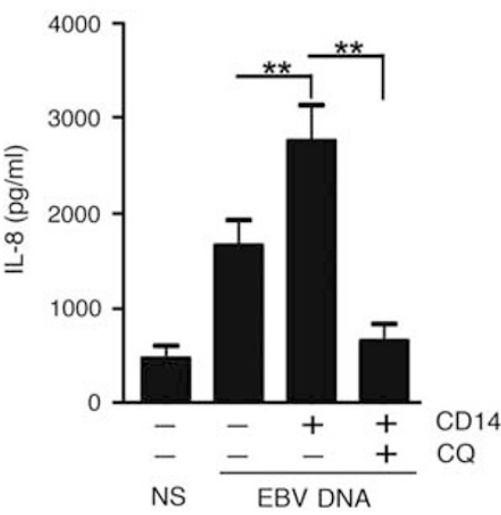

Figure 5 CD14 is essential for induction of IL-8 by EBV or EBV DNA. (a) dTHP-1 cells were transfected with CD14 expression vector or control vector for $48 \mathrm{~h}$. Cells were stimulated or not with infectious EBV $(\mathrm{MOI}=1.0)$ or $\mathrm{CpG}-2006(10 \mu \mathrm{g} / \mathrm{ml})$ for $10 \mathrm{~h}$, and then $/ \mathrm{L}-8$ mRNA levels were determined by qRTPCR. (b) dTHP-1 cells were transfected with either scrambled or CD14 siRNAs. Forty-eight hours post transfection, the knockdown efficiency of siRNA for CD14 was evaluated by western blotting. GAPDH was used as loading control. (c) dTHP-1 cells transfected with scrambled or CD14 siRNA were treated with EBV $(\mathrm{MOI}=1.0)$ or $\mathrm{CpG}-2006(10 \mu \mathrm{g} / \mathrm{ml})$. Ten hours post treatment, total RNA was extracted and $I L-8$ mRNA levels were determined by qRT-PCR. (d) dTHP-1 cells with transfection of CD14 expression vector were treated with EBV DNA. The IL-8 expression and secretion were determined by qRT-PCR (left panel) and ELISA (right panel). As indicated, some cells were also pretreated with CQ $(20 \mu \mathrm{M})$ for 30 min, before stimulation of EBV DNA. ${ }^{*} P<0.05 ; * * P<0.01$, compared with respective samples as indicated. NS, not stimulated.

Figure 6 LF suppresses the induction of IL-8, through inhibiting the binding of TLR9 to CD14. (a) Co-immunoprecipitation of LF with CD14. dTHP-1 cells were treated with hLF $(2.5 \mu \mathrm{g} / \mathrm{ml})$ for $1 \mathrm{~h}$ and the cell lysates were subjected to co-immunoprecipitation with either anti-CD14 or anti-LF antibodies (an unrelated IgG antibody was used as negative immunoprecipitation control). Immunoprecipitates were analyzed by western blot with anti-LF or antiCD14 antibodies. (b) dTHP-1 cells were transfected with LF and CD14 expression vectors, followed by treatment of EBV DNA or CpG-2006 (10 $\mu \mathrm{g} / \mathrm{ml})$ for $10 \mathrm{~h}$. Total RNA was harvested for IL-8 determinations by qRT-PCR. (c) dTHP-1 cells were transfected with CD14 expression vector, and then treated or not with LF $(50 \mu \mathrm{g} / \mathrm{ml})$ or CQ $(20 \mu \mathrm{M})$ for $1 \mathrm{~h}$, followed by EBV DNA stimulation. Ten hours post stimulation, total RNA was extracted and IL-8 mRNA levels were determined by QRT-PCR. (d) CD14 expression vector was transfected into the dTHP-1 cells with LF expression vector or deletion mutant LF vector (cannot bind to CD14). Forty-eight hours post transfection, the cells were stimulated with EBV DNA (10 $\mu \mathrm{g} / \mathrm{ml})$ or CpG-2006 (10 $\mu \mathrm{g} / \mathrm{ml})$ for $10 \mathrm{~h}$, and the IL-8 mRNA and protein expression levels were determined by qRT-PCR (left panel) and ELISA (right panel). ${ }^{*} P<0.05$; ${ }^{* *} P<0.01$, compared with respective samples as indicated. (e) dTHP-1 cells were treated or not with hLF (50 $\mu \mathrm{g} / \mathrm{ml}$ ) for $1 \mathrm{~h}$, followed by stimulation of EBV (MOI. 1.0$)$, or CpG-2006 $(10 \mu \mathrm{g} / \mathrm{ml})$ for 10 or $20 \mathrm{~min}$. The colocalization of TLR9 and CD14 was visualized by confocal microscopy using double labeling of cells with anti-TLR9 (Cy-5, red) and anti-CD14 (FITC, green) antibodies. (f) dTHP-1 cells were treated with hLF $(50 \mu \mathrm{g} / \mathrm{ml})$ or not. Then, the cells were stimulated with EBV DNA $(10 \mu \mathrm{g} / \mathrm{ml})$ or $\mathrm{CpG}-2006(10 \mu \mathrm{g} / \mathrm{ml})$. After $20 \mathrm{~min}$, the cells were collected, and the co-immunoprecipitation experiment was performed with antiCD14 antibodies or TLR9 antibodies. Immunoprecipitates were analyzed by western blot with anti-TLR9 or anti-CD14 antibodies. NS, not stimulated. 


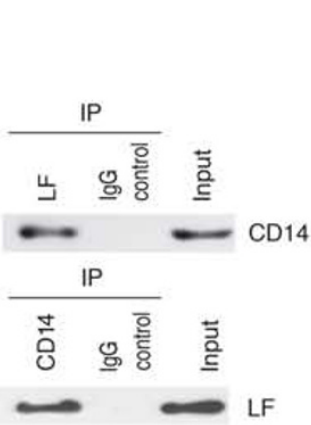

b

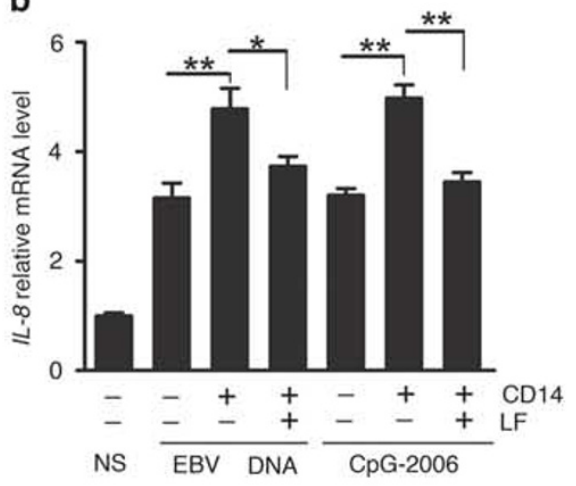

c

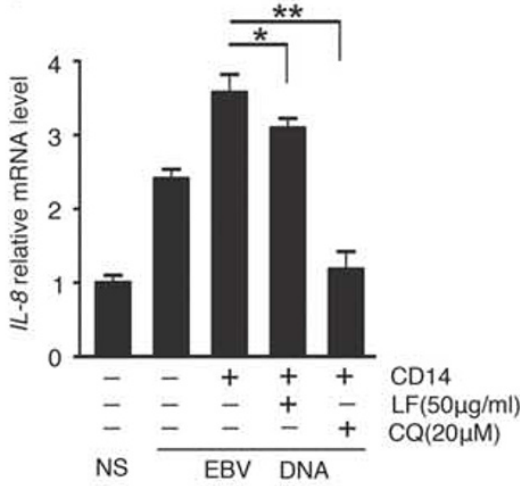

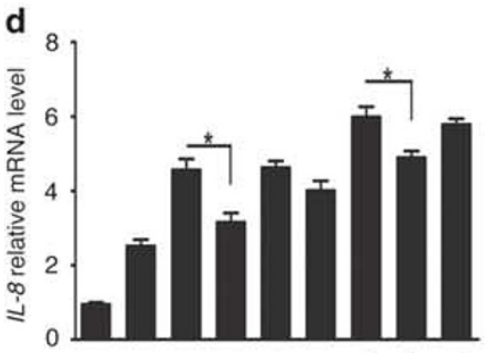

$-\ldots+++-+++$

$--+\ldots+-$

${ }_{-}+\ldots++\ldots+\ldots$ deletion mutant of $\mathrm{LF}$

NS $\overline{\text { EBV DNA }}$ CpG-2006

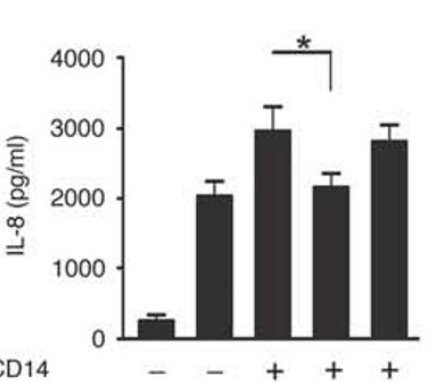

CD14

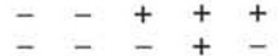

NS
EBV DNA

f

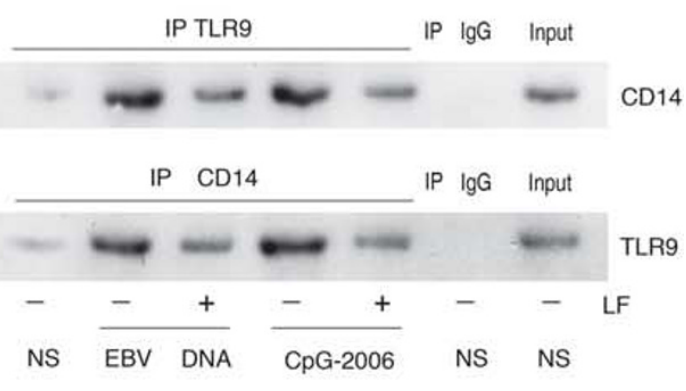

e
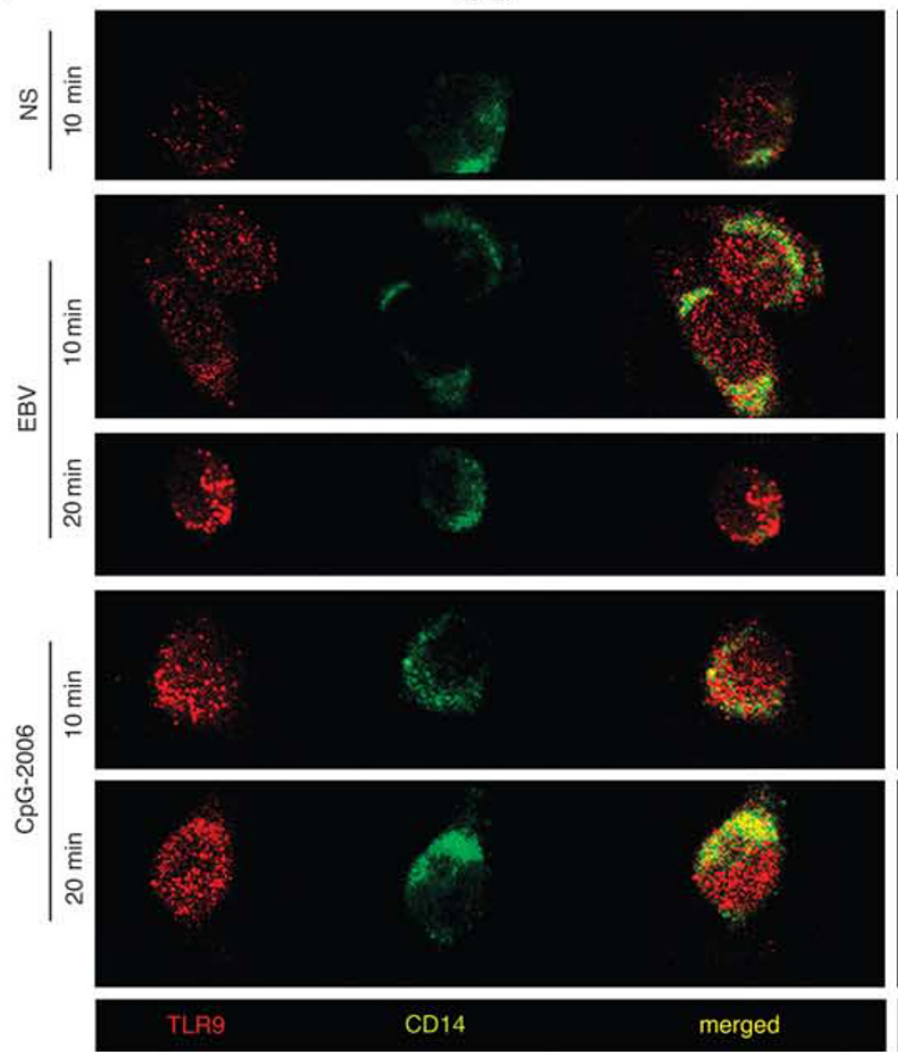

$\operatorname{LF}(50 \mu \mathrm{g} / \mathrm{ml})$
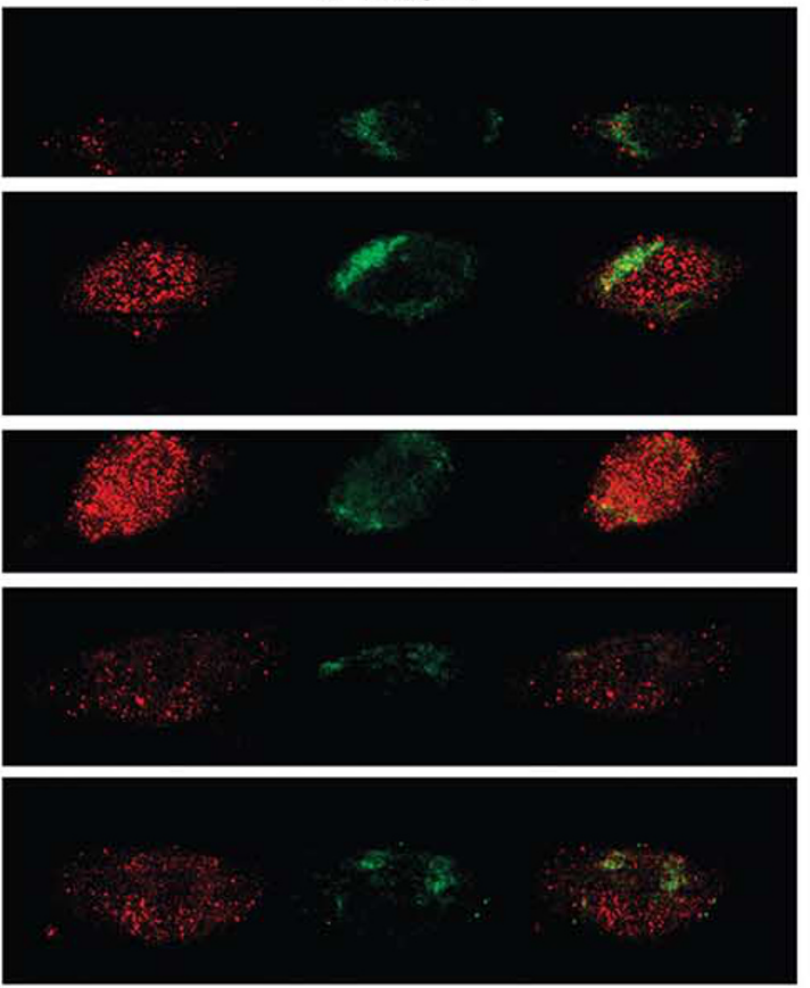

TLR9

CD14

merged

Figure 6 For caption please refer page 1195. 

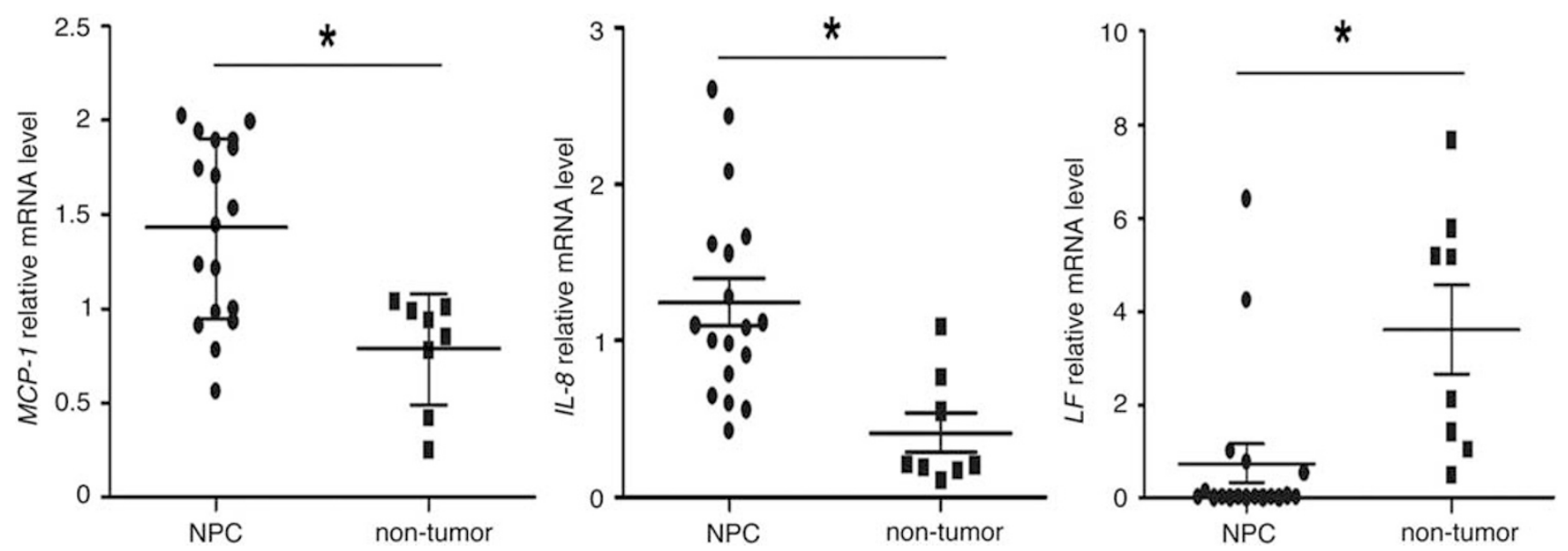

Figure $7 L F$ expression levels correlated inversely with $M C P-1$ and $I L-8$ in clinic specimens. The relative mRNA levels of $M C P-1, I L-8$, and $L F$ were determined by qRT-PCR in nasopharyngeal carcinoma tissues (NPC, $n=18$ ) and non-tumor nasopharyngeal epithelial tissues $(n=8)$. The expression levels were normalized to GAPDH. Data plotted represent expression levels in each subset from NPC $(\bullet)$ and non-tumor tissues $(\boldsymbol{\square})$. Bars represent the median. ${ }^{*} P<0.05$.

Table 2 Pairwise association between mRNA expression levels of $L F$ and $M C P-1$ or IL-8 for samples from NPC tissues $(n=18)$ and non-tumor nasophayngeal epithelial tissues $(n=8)$

$\begin{array}{cc}\begin{array}{c}M C P-1 \\ \text { level }\end{array} & \text { IL-8 mRNA } \\ \text { level }\end{array}$

\begin{tabular}{lrr} 
LF mRNA level & & \\
Spearman correlation coefficient & -0.577 & -0.541 \\
Significance (two-tailed) & 0.002 & 0.004 \\
\hline
\end{tabular}

Correlation is significant at the 0.05 level (two-tailed).

results indicate that LF may negatively regulate TLR9 activation through binding to its co-receptor, CD14, and interfering with the TLR9-CD14 interaction.

\section{LF Expression levels Correlated Inversely with MCP-1 and IL-8 Expression in NPC Tissues}

Inflammation is an important factor for tumorgenesis, and inflammatory cytokines IL-8 and MCP-1 have important roles in NPC. Our previous studies showed that LF is a tumor-suppressor gene for NPC, which is downregulated in NPC tissue. We speculated that the inhibitory effects of LF on the secretion of IL- 8 and MCP-1 may be an important mechanism for its tumor-suppressing function. Therefore, we used qRT-PCR to assay $L F, I L-8$, and $M C P-1$ expression levels in NPC tissues $(n=18)$ or non-tumor nasopharyngeal epithelial tissues $(n=8)$ to define the anti-inflammatory roles of LF in the tumorgenesis of NPC. LF mRNA expression levels were significantly downregulated in NPC tissues compared with non-tumor tissues, whereas $I L-8$ and $M C P-1$ were increased in NPC tissues (Figure 7). Spearman's correlation test was used to evaluate the pairwise association of the samples' $L F$ and $I L-8$ or MCP-1 levels. The pairwise associations between $L F$ levels and $I L-8$ (Spearman correlation coefficient $r=-0.541, P=0.004$ ) or $M C P-1$ (Spearman correlation coefficient $r=-0.577, P=0.002$ ) are shown in Table 2.

Our findings demonstrate that LF has properties consistent with anti-inflammation function. Ability of LF to reduce IL-8 and MCP-1 expression induced by EBV infection may be dependent on interfering with the pattern recognition of TLR2 and TLR9.

\section{DISCUSSION}

A wide range of functions has now been described for LF, including iron homeostasis, anti-microbial activity, antiinflammatory activity, and against protection cancer. ${ }^{13-15} \mathrm{LF}$ displays anti-tumor and anti-metastatic activity in different types of cancer. ${ }^{24}$ We previously observed a notable reduction of LF in NPC tissues and identified it as a candidate tumor suppressor for NPC. Our subsequent studies showed that LF inhibits NPC cell proliferation, induces cell cycle $G_{1} / S$ arrest, modulating MAPK signaling pathway, and cyclin D1-related proteins ${ }^{20}$ and inhibits NPC metastasis by suppressing AKT activity. ${ }^{25}$

EBV is widespread in human populations, and EBV infection has been linked to the tumorigenesis of multiple cancers including NPC. ${ }^{1}$ One important consequence of EBV infection is malignant transformation of epithelial cells, promoted by EBV-encoded protein and microRNAs. ${ }^{26-29}$ Another is EBV-induced inflammatory cytokines in the tumor microenvironment, resulting in angiogenesis and metastasis. ${ }^{8}$ As a key component of innate immunity, LF has vital roles in host defense against infection and excessive inflammation. We recently reported that LF protects both 
primary B cells and epithelial cells from EBV infection and its protective role may be one of its activities as a tumor suppressor in NPC. ${ }^{21}$ However, besides its role in preventing EBV infection, does LF also have anti-inflammatory activity against the EBV-induced inflammatory responses?

It has been widely reported that LF has inhibitory effects on inflammatory responses. ${ }^{16,18}$ In the present study, we first found that LF can inhibit EBV-induced IL-8 and MCP-1 expression in macrophages. Further, by means of qRT-PCR, we found that LF mRNA is absent or downregulated in most NPC tissues and its expression levels are negatively correlated with IL-8 or MCP-1 levels. The chemokine IL-8, initially identified as a neutrophil-activating and chemotactic factor, has multiple roles as a proinflammatory cytokine by mediating the activation and chemotaxis of various immune cell types, and leads to chronic inflammatory conditions if aberrantly expressed. ${ }^{30,31}$ Many types of human tumors, including breast, colon, cervical, gastric, lung, and ovarian cancers, express high levels of IL-8 relative to normal tissues. ${ }^{32}$ IL-8 participates in tumorigenesis by promoting the proliferation, survival, and migration of tumor cells, and recruiting neutrophils to the site of the tumor. ${ }^{32} \mathrm{MCP}-1$, also known as chemokine (C-C motif) ligand 2 (CCL2), is a highly potent chemoattractant of monocytes/macrophages to sites of inflammation as well as tumor sites. ${ }^{33}$ Multiple human tumors have also been showed to express high levels of MCP-1, ${ }^{34-36}$ and MCP-1 could promote tumor progression and metastasis as well. ${ }^{37-39}$

The immune cells, such as monocytes/macrophages and dendritic cells, are important constitutes for the microenvironment. ${ }^{10}$ These cells rapidly recognize a wide variety of molecules expressed by pathogens, such as EBV. Several TLRs, including TLR2, TLR3, and TLR9, participate in recognition of EBV by monocytes/macrophages and dendritic cells. $5,7,11,12$

Gaudreault et $a l^{7}$ revealed that stimulation of monocytes with EBV virions leads to the release of MCP-1 and IL-8 through the activation of TLR2. ${ }^{7}$ TLR2 recognizes glycoproteins of the viral envelope, resulting in the activation of $\mathrm{NF}-\kappa \mathrm{B}$ signaling and production of these inflammatory cytokines. $^{7}$ LF is a glycoprotein with a large net positive charge, which enables it to bind to numerous molecules. Our previous study demonstrated that LF could perturb the binding of EBV to its receptor CD21 on the B-cell surface. ${ }^{21}$ Here, we speculated that LF could bind to TLR2, subsequently weakening the recognition ability of TLR2 to EBV virions and suppressing the activation of TLR2. Confocal microscopy and the co-immunoprecipitation assay indicated that LF can interact with TLR2. We also used luciferase reporter assays to demonstrate that LF does repress NF- $\kappa B$ activation. These findings suggest that LF is likely to interfere with EBV binding to TLR2 and to prevent the activation of TLR2 signaling.

Besides the recognition of EBV virions by cell surface TLR2, a second TLR, TLR9, may contribute to the secretion of inflammatory cytokine IL- 8 by monocytes, through recognition of viral DNA. ${ }^{5}$ We observed that LF could repress the EBV-DNA-induced IL-8 expression in macrophages, which implies that LF might interfere with the engagement and activation of TLR9. Confocal microscopy experiments showed that LF pretreatment represses the redistribution of TLR9 in late vesicles, which indicates that LF could inhibit the activation of TLR9. Recently, CD14 was reported to be indispensable for TLR9-dependent induction of proinflammatory cytokines by viruses. ${ }^{23}$ In an attempt to evaluate whether CD14 is involved in TLR9 activation by EBV, we observed that CD14 seems to be indispensable for EBV-mediated TLR9 activation. In fact, induction of IL-8 expression by both EBV virions and EBV-DNA stimulation in macrophages was enhanced after transfection of the CD14 plasmid, whereas the induction is abolished by CD14 knockdown. LF is well known to be a potential antiinflammatory molecule for its ability of binding to CD14 and inhibiting endotoxin-mediated inflammatory response. ${ }^{17} \mathrm{We}$ therefore proposed that the interaction of LF and CD14 is linked to the inhibitory effect of LF on EBV-DNA-induced TLR9 activation. Our results showed that LF binds to CD14, and partially inhibits the effects of CD14 on promoting EBV DNA-induced inflammation as well as IL-8 upregulation and secretion, whereas deletion mutant of LF, which cannot bind to CD14, does not have this ability. We further confirmed that LF inhibits the TLR9 activation by means of interfering CD14 binding to TLR9, which will inhibit the ability of TLR9 recognizing EBV DNA. Therefore, the ability of LF to interfere with binding of CD14 and TLR9 may account for its inhibitory effects on EBV DNA-induced and TLR9-dependent inflammatory response.

As a key protein of innate immunity system, LF exhibits inhibitory roles against a wide variety of viruses. We first report that LF can prevent from EBV infection, and also inhibit EBV-induced inflammation. LF's ability of inhibiting EBV infection is linked to its surface positive charge, which enables it to bind to many macromolecules. As LF shows inhibitory potential in EBV infection, LF may be applied in preventative medicine or nutrition supplies for EBV infection-related diseases.

\section{ACKNOWLEDGMENTS}

This work was supported by National Nature Scientific Foundation of China (81171988, 91229122, 81272975), China 111 Project (No. 111-2-12), Ministry of Education (NCET-11-0520, SRF for ROCS), and Hunan Province Natural Sciences Foundation of China (14JJ2022).

\section{DISCLOSURE/CONFLICT OF INTEREST}

The authors declare no conflict of interest.

1. Young LS, Rickinson AB. Epstein-Barr virus: 40 years on. Nat Rev Cancer 2004;4:757-768.

2. Nakamura $\mathrm{H}$, Iwakiri $\mathrm{D}$, Ono $\mathrm{Y}$, et al. Epstein-Barr-virus-infected human T-cell line with a unique pattern of viral-gene expression. Int J Cancer 1998;76:587-594. 
3. Savard $M$, Belanger $C$, Tardif $M$, et al. Infection of primary human monocytes by Epstein-Barr virus. J Virol 2000;74:2612-2619.

4. Savard M, Gosselin J. Epstein-Barr virus immunossuppression of innate immunity mediated by phagocytes. Virus Res 2006;119:134-145.

5. Fiola $\mathrm{S}$, Gosselin D, Takada $\mathrm{K}$, et al. TLR9 contributes to the recognition of EBV by primary monocytes and plasmacytoid dendritic cells. J Immunol 2010;185:3620-3631.

6. Gosselin J, Flamand L, D'Addario M, et al. Infection of peripheral blood mononuclear cells by herpes simplex and Epstein-Barr viruses. Differential induction of interleukin 6 and tumor necrosis factoralpha. J Clin Invest 1992;89:1849-1856.

7. Gaudreault E, Fiola S, Olivier M, et al. Epstein-Barr virus induces MCP-1 secretion by human monocytes via TLR2. J Virol 2007;81:8016-8024.

8. Coussens LM, Werb Z. Inflammation and cancer. Nature 2002;420: 860-867.

9. Tugizov $S$, Herrera $R$, Veluppillai $P$, et al. Epstein-Barr virus (EBV)infected monocytes facilitate dissemination of EBV within the oral mucosal epithelium. J Virol 2007:81:5484-5496.

10. Akira $S$, Uematsu $S$, Takeuchi $O$. Pathogen recognition and innate immunity. Cell 2006;124:783-801.

11. Lund J, Sato A, Akira S, et al. Toll-like receptor 9-mediated recognition of Herpes simplex virus-2 by plasmacytoid dendritic cells. J Exp Med 2003;198:513-520.

12. Iwakiri $D$, Zhou L, Samanta $M$, et al. Epstein-Barr virus (EBV)-encoded small RNA is released from EBV-infected cells and activates signaling from Toll-like receptor 3. J Exp Med 2009;206:2091-2099.

13. Legrand $D$, Mazurier J. A critical review of the roles of host lactoferrin in immunity. Biometals 2010;23:365-376.

14. Teng CT. Lactoferrin: the path from protein to gene. Biometals 2010;23:359-364

15. Gonzalez-Chavez SA, Arevalo-Gallegos S, Rascon-Cruz Q. Lactoferrin: structure, function and applications. Int J Antimicrob Agents 2009; 33:301e301-308.

16. Elass-Rochard E, Legrand D, Salmon V, et al. Lactoferrin inhibits the endotoxin interaction with CD14 by competition with the lipopolysaccharide-binding protein. Infect Immun 1998;66:486-491.

17. Baveye $\mathrm{S}$, Elass E, Fernig DG, et al. Human lactoferrin interacts with soluble CD14 and inhibits expression of endothelial adhesion molecules, E-selectin and ICAM-1, induced by the CD14-lipopolysaccharide complex. Infect Immun 2000;68:6519-6525.

18. Britigan $\mathrm{BE}$, Lewis TS, Waldschmidt $\mathrm{M}$, et al. Lactoferrin binds CpG containing oligonucleotides and inhibits their immunostimulatory effects on human B cells. J Immunol 2001;167:2921-2928.

19. Xiong $\mathrm{W}$, Zeng $\mathrm{ZY}$, Xia $\mathrm{JH}$, et al. A susceptibility locus at chromosome 3 p21 linked to familial nasopharyngeal carcinoma. Cancer Res 2004:64:1972-1974.

20. Zhou Y, Zeng Z, Zhang W, et al. Lactotransferrin: a candidate tumor suppressor-Deficient expression in human nasopharyngeal carcinoma and inhibition of NPC cell proliferation by modulating the mitogenactivated protein kinase pathway. Int J Cancer 2008;123:2065-2072.

21. Zheng $Y$, Zhang $W, Y e ~ Q$, et al. Inhibition of Epstein-Barr virus infection by lactoferrin. J Innate Immun 2012;4:387-398.

22. Vollmer J, Krieg AM. Immunotherapeutic applications of CpG oligodeoxynucleotide TLR9 agonists. Adv Drug Deliv Rev 2009;61:195-204.
23. Baumann $\mathrm{CL}$, Aspalter IM, Sharif O, et al. CD14 is a coreceptor of Tolllike receptors 7 and 9. J Exp Med 2010;207:2689-2701.

24. Rodrigues L, Teixeira J, Schmitt F, et al. Lactoferrin and cancer disease prevention. Crit Rev Food Sci Nutr 2009;49:203-217.

25. Deng $M$, Zhang $W$, Tang $H_{\text {, et }}$ al. Lactotransferrin acts as a tumor suppressor in nasopharyngeal carcinoma by repressing AKT through multiple mechanisms. Oncogene 2013;32:4273-4283.

26. Dawson CW, Port RJ, Young LS. The role of the EBV-encoded latent membrane proteins LMP1 and LMP2 in the pathogenesis of nasopharyngeal carcinoma (NPC). Semin Cancer Biol 2012;22: 144-153.

27. Yoshizaki T, Kondo S, Wakisaka N, et al. Pathogenic role of Epstein-Barr virus latent membrane protein-1 in the development of nasopharyngeal carcinoma. Cancer Lett 2013;337:1-7.

28. Lei T, Yuen KS, Xu R, et al. Targeting of DICE1 tumor suppressor by Epstein-Barr virus-encoded miR-BART3* microRNA in nasopharyngeal carcinoma. Int J Cancer 2013;133:79-87.

29. Ye Y, Zhou $Y$, Zhang $L$, et al. EBV-miR-BART1 is involved in regulating metabolism-associated genes in nasopharyngeal carcinoma. Biochem Biophys Res Commun 2013;436:19-24.

30. Yoshimura T, Matsushima K, Oppenheim JJ, et al. Neutrophil chemotactic factor produced by lipopolysaccharide (LPS)-stimulated human blood mononuclear leukocytes: partial characterization and separation from interleukin 1 (IL 1). J Immunol 1987;139: 788-793.

31. Skov L, Beurskens FJ, Zachariae CO, et al. IL-8 as antibody therapeutic target in inflammatory diseases: reduction of clinical activity in palmoplantar pustulosis. J Immunol 2008;181:669-679.

32. Waugh DJ, Wilson C. The interleukin-8 pathway in cancer. Clin Cancer Res 2008;14:6735-6741.

33. Lee HW, Choi HJ, Ha SJ, et al. Recruitment of monocytes/macrophages in different tumor microenvironments. Biochim Biophys Acta 2013;1835:170-179.

34. Negus RP, Stamp GW, Relf MG, et al. The detection and localization of monocyte chemoattractant protein-1 (MCP-1) in human ovarian cancer. J Clin Invest 1995;95:2391-2396.

35. Graves DT, Barnhill R, Galanopoulos T, et al. Expression of monocyte chemotactic protein-1 in human melanoma in vivo. Am J Pathol 1992;140:9-14.

36. Riethdorf $L$, Riethdorf S, Gutzlaff K, et al. Differential expression of the monocyte chemoattractant protein-1 gene in human papillomavirus16-infected squamous intraepithelial lesions and squamous cell carcinomas of the cervix uteri. Am J Pathol 1996;149:1469-1476.

37. Ueno $T$, Toi $M$, Saji $H$, et al. Significance of macrophage chemoattractant protein-1 in macrophage recruitment, angiogenesis, and survival in human breast cancer. Clin Cancer Res 2000;6: 3282-3289.

38. Arenberg DA, Keane MP, DiGiovine $B$, et al. Macrophage infiltration in human non-small-cell lung cancer: the role of CC chemokines. Cancer Immunol Immunother 2000;49:63-70.

39. Ohta M, Kitadai Y, Tanaka S, et al. Monocyte chemoattractant protein1 expression correlates with macrophage infiltration and tumor vascularity in human esophageal squamous cell carcinomas. Int J Cancer 2002;102:220-224. 